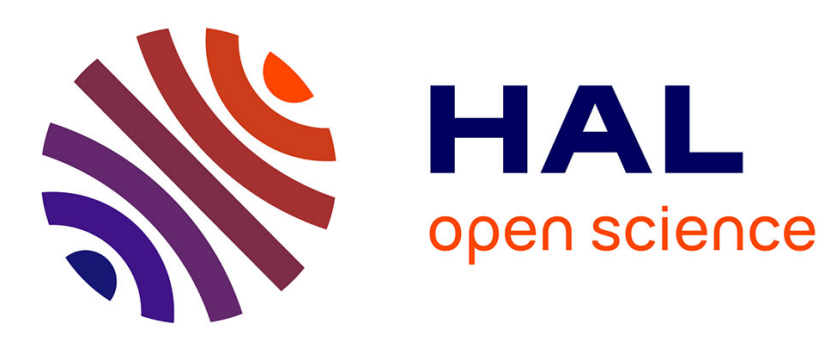

\title{
Mechanical behavior of annulus fibrosus: a microstructural model of fibers reorientation.
}

Dominique Ambard, Fabien Cherblanc

\section{To cite this version:}

Dominique Ambard, Fabien Cherblanc. Mechanical behavior of annulus fibrosus: a microstructural model of fibers reorientation.. Annals of Biomedical Engineering, 2009, 37 (11), pp.2256-2265. 10.1007/s10439-009-9761-7 . hal-00449672

\section{HAL Id: hal-00449672 \\ https://hal.science/hal-00449672}

Submitted on 3 Feb 2011

HAL is a multi-disciplinary open access archive for the deposit and dissemination of scientific research documents, whether they are published or not. The documents may come from teaching and research institutions in France or abroad, or from public or private research centers.
L'archive ouverte pluridisciplinaire HAL, est destinée au dépôt et à la diffusion de documents scientifiques de niveau recherche, publiés ou non, émanant des établissements d'enseignement et de recherche français ou étrangers, des laboratoires publics ou privés. 
Mechanical behavior of annulus fibrosus: a microstructural model of fibers reorientation.

\author{
D. Ambard, F. Cherblanc \\ LMGC, UMR CNRS 5508, University of Montpellier 2 \\ Department of Mechanics and Civil Engineering \\ 34095 Montpellier cedex 5, France \\ Tel : +33(0)467144680 \\ Fax $:+33(0) 467144555$ \\ dominique.ambard@univ-montp2.fr
}




\begin{abstract}
Experimental uniaxial tensile tests have been carried out on annulus tissue samples harvested on pig and lamb lumbar intervertebral discs. When subjecting the samples to loading cycles, the stress-strain curves exhibit strong non-linearities and hysteresis. This particular behavior results from the anisotropic microstructure of annulus tissue composed of woven oriented collagen fibers embedded in the extracellular matrix. During uniaxial tension, the collagen fibers reorient toward the loading direction increasing its global stiffness. To describe this behavior, we propose an heuristic two-dimensional rheological model based on three mechanical and one geometrical characteristics. The latter one is the fibers orientation angle becoming the key parameter that govern the macroscopic mechanical behavior. The experimental results are used to identify the physical properties associated with the rheological model, leading to an accurate representation of the stress-strain curve over a complete loading cycle. In this framework, the fibers reorientation can solely account for the rigidity increase while the hysteresis is associated with liquid viscous flows through the matrix. Based on this representation, unusual coupling effects between strains and fluid flows can be observed, that would significantly affect the cell nutrients transport mechanisms. Keywords : intervertebral disc, annulus fibrosus, fibers reorientation, microstructural model, mechanical behavior.
\end{abstract}




\section{Introduction}

The intervertebral disc is a highly-specialized element of the spine that provides flexibility and adsorbing capacities. When mechanical loads are transmitted along the spine, the intervertebral disc mainly supports compression and flexion stresses. This results in a hydrostatic excessive pressure in the nucleus pulposus and generates circumferential tensile stresses in the surrounding annulus fibrosus. Maximal physiologic strains observed along the annulus superficial tissue are about $4 \%$ when the disc is in compression or torsion, and about $6 \%$ when the disc is in flexion or exten$\operatorname{sion}^{3,7}$, but never exceed $10 \%{ }^{27,7}$. To hold these large circumferential strains, the annulus tissue is composed of a woven oriented structure of collagen fibers embedded in a highly hydrated matrix. This structured layout provides some interesting mechanical properties, indeed, an anisotropic and non-linear behavior is observed when subjected to uniaxial tensile tests ${ }^{11,23,8,31,32,15,20,14,29}$.

Using fiber-reinforced continuum theory, hyperelastic formulations have been developed to account for this non-linear behavior. This leads to some exponential strain energy functions able to curvefit the experimental stress-strain behaviors from various configurations: tension or compression on axial, radial or circumferential samples ${ }^{23}$. Some attempts to investigate the contributions of individual structural components (matrix, fibers, fiber-matrix or fiber-fiber interactions) have been done ${ }^{14}$. Nevertheless, with these homogenized approaches, the geometrical modifications of the micro-structure are hidden in the strain energy functions, what make tough to identify the micro-structural origin of the annulus fibrosus complex behavior. Actually, when subjected to uniaxial tension, the collagen fibers reorient toward the loading direction. With techniques of digital image processing, this reorientation has been quantified showing significant geometrical modifications of the micro-structure ${ }^{31,13}$. These experimental observations imply that the fibers orientation variations could be large enough to induce a macroscopic stiffness increase. This point of view is confirmed by biaxial tests that point out higher stiffnesses but less non-linearities ${ }^{3,5}$. Therefore, the fibers orientation could be the key parameter that governs the mechanical behavior of annulus and this geometrical characteristic could be sufficient to account for the material properties differences between outer and inner annulus tissue.

Concerning the biological processes, the intervertebral disc is an avascular organ; it means that the cell nutrients are transferred from the upper and lower vertebrae through the disc by advective or diffusive mechanisms ${ }^{12,35}$. Resulting from daily loading cycles on the disc, extracellular fluid displacements increase mass exchange between outer and inner disc regions and participate to the nutrients transport. These liquid flows could be modified by tissue mechanical strains ${ }^{10,30}$ or flow characteristics of the cartilaginous end-plates ${ }^{1}$. In this framework, the contribution played by the 
mechanical behavior of the annulus tissue has not been clearly established. Since the annulus fibrosus is highly hydrated, the liquid phase plays a significant role in its mechanical behavior. Some viscous effects are observed resulting from fluid filtration through the matrix and leads to hysteretic behaviors. Despite the fact that the intervertebral disc is subjected to daily loading cycles, the annulus response to cycling tensile test has not been much investigated.

In saturated porous media, the coupling effects between strains and fluid transfers are usually taken into account using a poro-elastic approach ${ }^{4,2,24}$. In such formulations, the skeleton Poisson's coefficient that governs the total volume change rate is of the highest importance. When dealing with lengthy fibers composite materials, an anisotropic behavior is observed. By relying on an homogeneous material point of view, the measured macroscopic Poisson's coefficients can be greater than the classical upper limit given by 0.5 . In such cases, the total volume variation resulting from uniaxial tensile stresses can be negative depending on the fibers orientation. If the annulus tissue behaves as such composite materials, some non-usual coupling effects between mechanical behavior and fluid transfers can be observed. Its analysis and description is a major issue in order to develop a comprehensive model of the intervertebral disc.

The goal of this study is to develop a simple heuristic model able to describe the homogenized mechanical behavior (non-linearity, hysteresis) and to retrieve micro-structural characteristics (fibers reorientation, coupling with the fluid phase). This model is based on simple rheological elements such as linear springs or dampers laid out in a geometrical configuration similar to the woven micro-structure of annulus. Therefore, macroscopic non-linearities are generated by geometrical reorientation of fibers during stretching. This description underlines that the nonlinear mechanical behavior of annulus tissue mainly results from its particular woven architecture rather than from micro-scale nonlinearities. The characteristics of this model are identified using some experimental uniaxial tensile tests realized on pig and lamb annulus samples. Eventually, the mechanical behavior is discussed in terms of cyclic effects.

\section{Microstructure model}

The microstructure of annulus fibrosus is composed of collagen fibers embedded in the extracellular matrix. This matrix is mainly composed of water and elastin fibers which perform a junction between collagen fibers ${ }^{37}$. The collagen fibers have a relatively large tensile ultimate strength while being flexible, they confer the softness and resistance to this biological tissue. The secondary network of elastin fibers allows to preserve the lateral micro-displacement of collagen fibers. The collagen fibers are oriented with an angle $\theta_{0}$ with respect to the horizontal plane. This angle, $\theta_{0}$, 


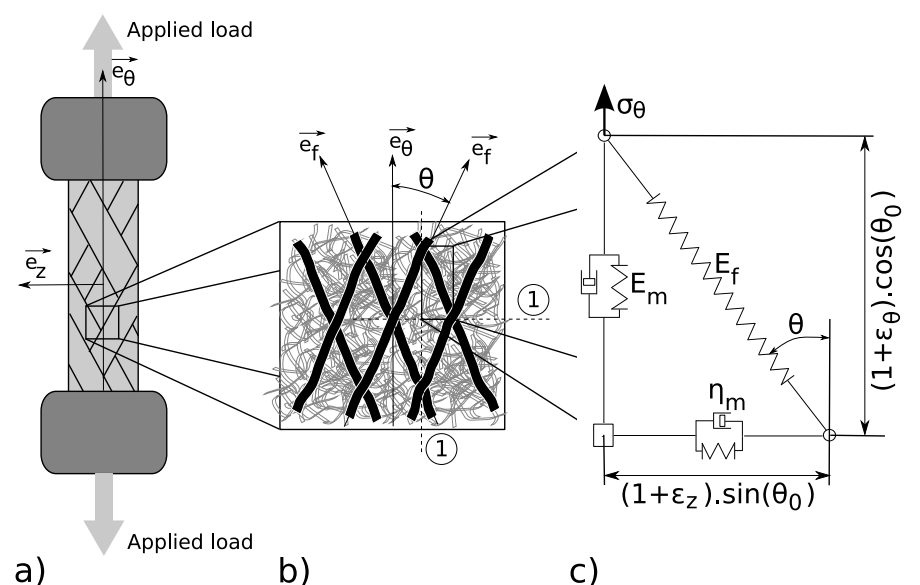

Figure 1: a) Representation of one sample and loading direction. b) Description of internal microstructure of annulus fibrosus. c) Rheological model of annulus fibrosus microstructure.

generally ranges from $25^{\circ}$ to $45^{\circ}$. The annulus fibrosus is structured with concentric lamellae around the nucleus pulposus alternately oriented with an angle of $+\theta_{0}$ and $-\theta_{0}$.

When focusing on an experimental sample (Fig. 1a), the microstructure of the annulus tissue can be represented by a regular layout of collagen fibers embedded in an homogeneous matrix (Fig. 1b). Using the symmetry properties of this structure, a representative elementary volume (REV) is described by one collagen fiber interacting with a triangular zone of matrix (Fig. 1c). The collagen fiber is oriented with an initial angle, $\theta_{0}$. The fiber is supposed to have a linear elastic behavior characterized by an elastic modulus, $E_{f}$, which is a fair assumption when subjected to low strains ${ }^{15}$. The matrix composed of an isotropic mixture of elastin fibers saturated with physiological liquid is modeled through a visco-elastic behavior characterized by an elastic modulus, $E_{m}$, and a viscosity, $\eta_{m}$. Actually, the complete rheological model presented in Fig. (1c) should be assimilated to a mechanism since the orientation, $\theta$, changes during macroscopic stretching. Indeed, since the rheological behaviors introduced are linear (elastic and visco-elastic), macroscopic non-linearities will directly results from geometrical modifications imposed by the circumferential strain, $\varepsilon_{\theta}$. Thereby, the mechanical behavior of annulus fibrosus depends on 4 parameters: 3 mechanical parameters $\left(E_{m}, \eta_{m}, E_{f}\right)$ and 1 geometrical parameter $\theta_{0}$. This rheological model should be able to simulate both non-linear mechanical behavior and hysteresis over a loading cycle.

\section{Geometrical relations}

The geometrical orientation of collagen fibers, $\theta$, is related to the initial angle, $\theta_{0}$, through the mechanical strains (Eqs. 1 and 2). The first relation (Eq. 1) gives the reorientation as a function 
of the collagen fibers strain, $\varepsilon_{f}$, and the sample circumferential strain, $\varepsilon_{\theta}$, while the second one (Eq. 2) shows the dependence on the tansversal strain, $\varepsilon_{z}$.

$$
\begin{aligned}
& \cos (\theta)=\cos \left(\theta_{0}\right) \frac{1+\varepsilon_{\theta}}{1+\varepsilon_{f}} \\
& \sin (\theta)=\sin \left(\theta_{0}\right) \frac{1+\varepsilon_{z}}{1+\varepsilon_{f}}
\end{aligned}
$$

Experimental tensile tests are carried out at a constant velocity rate, $V$, for the length of the samples $h_{0}$ so that the circumferential strain, $\varepsilon_{\theta}$, is given by:

$$
\varepsilon_{\theta}=\frac{V}{h_{0}} t
$$

where $h_{0}$ is the initial length of an experimental sample $(\approx 10 \mathrm{~mm})$.

\section{Behavior laws}

Concerning the mechanical behavior constitutive laws, a linear elastic model is used for the collagen fibers (Eq. 4) and a visco-elastic model for the matrix (Eqs. 5 and 6). These relations depends on the 3 mechanical parameters $\left(E_{m}, \eta_{m}, E_{f}\right)$ introduced in the previous section.

$$
\begin{aligned}
& \sigma_{f}=E_{f} \varepsilon_{f} \\
& \sigma_{\theta m}=E_{m} \varepsilon_{\theta}+\eta_{m} \dot{\varepsilon}_{\theta} \\
& \sigma_{z m}=E_{m} \varepsilon_{z}+\eta_{m} \dot{\varepsilon}_{z}
\end{aligned}
$$

\section{Equilibrium equations}

By relying on the symmetry conditions associated with the rheological model described in Fig. (1), the corresponding mechanical equilibrium equations are given below:

$$
\begin{gathered}
\sigma_{\theta}=\sigma_{\theta m}+2 \sigma_{f} \cos \left(\theta_{0}\right) \frac{1+\varepsilon_{\theta}}{1+\varepsilon_{f}} \\
0=\sigma_{z m}-2 \sigma_{f} \sin \left(\theta_{0}\right) \frac{1+\varepsilon_{z}}{1+\varepsilon_{f}}
\end{gathered}
$$

\section{Numerical simulation and parameters identification}

This set of first-order non-linear differential equations (Eqs. 4 to 8 ) is solved using an implicit Euler's scheme associated with a Newton-Raphson's algorithm. The numerical time step is identical than for experimental tests, $d t=0.01 \mathrm{~s}$. The convergence of Newton-Raphson's procedure is fixed to $10^{-7} \mathrm{Mpa}$ with respect to the equilibrium conditions. 

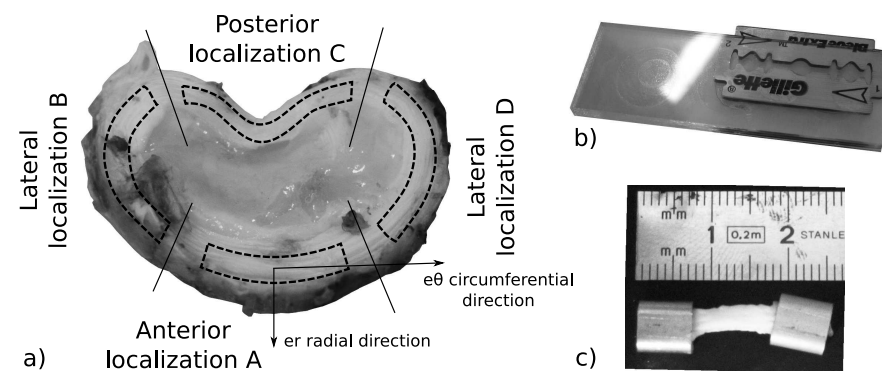

Figure 2: a) Lumbar disc with the specimens locations. b) Specific tool used for cutting rectangular specimen. c) Annulus specimen with aluminum ring.

To identify the 4 parameters $E_{m}, \eta_{m}, E_{f}$ and $\theta_{0}$ from the experimental data, a LevenbergMarquardt non-linear fitting algorithm has been used (Python Scipy library). The quality of the rheological model is evaluated by means of the correlation coefficient $R^{2}$.

\section{Materials and method}

\section{Specimen preparation}

Two lumbar discs (L3-L4) were harvested from cadaver of lambs. These lambs were 6 months old and obtained from the veterinary school of Lyon, France. To avoid a blood-clot formation into the cartilage end-plates, a subcutaneous injection of heparin (Innohep 4500UI, tinzaparine, Leo Pharma) was administered the day before the sacrifice. Under general anesthesia and appropriate analgesia, the spinal segment (L3-L4) was removed. This spinal segment was stored into a $10 \%$ DMSO solution (Dimethyl sulfoxide, a cryoprotectant) in a $-12^{\circ} \mathrm{C}$ freezer. Associated to this lumbar disc, four lumbar discs (L1-L5) were harvested from the cadaver of a domestic pig (160 $\mathrm{Kg}$ ) obtained in a local slaughter house. This lumbar spine was excised and the specimens were also stored in a $-12^{\circ} \mathrm{C}$ freezer.

The discs were separated from the vertebral bodies by blunt dissection. Next, the posterior, lateral and anterior quadrants were separated from each disc using a surgical knife. In brief, all specimens were excised in anatomical cut parallel to the horizontal plane along the circumferential direction $\overrightarrow{e_{\theta}}$. From each quadrant (Fig. 2a), a subsample of biological tissue was extracted to determine the solid mass fraction. It is evaluated by differential weighing with a high-precision balance (Sartorius) after 24 hours drying at $105^{\circ}$. Some significant differences in composition between the pig and lamb specimens have been observed, revealed by the statistical $t$-test $(p=$ 0.0027) (Fig. 3b). 

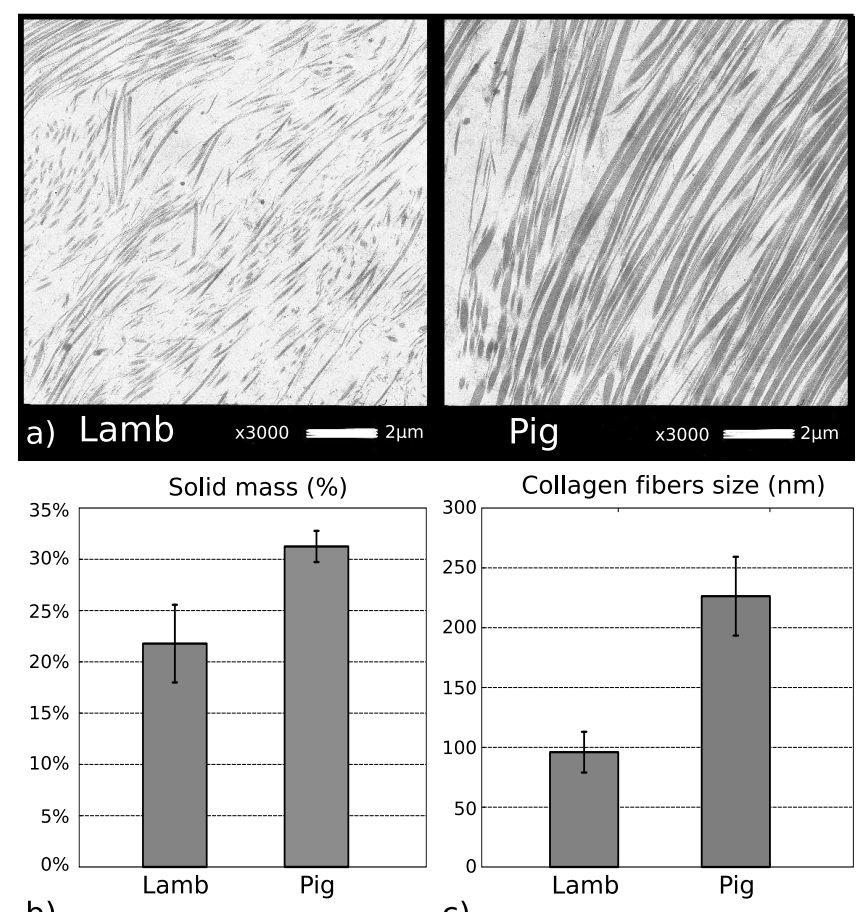

b)

c)

Figure 3: a) Transmission electronic microscopy images of a lamb and a pig specimen. White regions correspond to the matrix and black ones to the collagen fibers. b) Solid mass fractions of lamb and pig specimens. c) Collagen fibers size for lamb and pig specimens measured with ImageJ. 

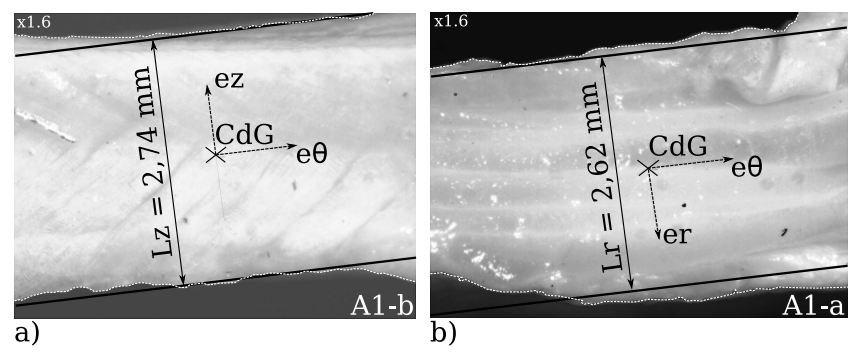

Figure 4: Numerical threshold technique. a) Specimen size evaluated in the plane $\left(\overrightarrow{e_{\theta}}, \overrightarrow{e_{z}}\right)$. b) Specimen size evaluated in the plane $\left(\overrightarrow{e_{\theta}}, \overrightarrow{e_{r}}\right)$.

Another subsample of each quadrant has been analyzed by means of transmission electron microscopy (Fig. 3a). Using the same magnification (x3000), the microscopic analysis has shown a higher matrix fraction with lamb specimens than with pig ones. This confirms the global measures obtained by weighing technique (Fig. 3b). This microscopic analysis has also underlined a significant difference $\left(p=2 \times 10^{-7}\right)$ between the collagen fibers mean size of lamb and pig (Fig. 3c).

\section{Specimen preparation for mechanical testing}

From each quadrant (Fig. 2a), one plane-parallel specimen was extracted using a specific tool. This tool was realized with two razor blades separated with a $2 \mathrm{~mm}$-spacer. The length of each specimen $h_{0}$ was limited using a surgical knife at approximately $10 \mathrm{~mm}$. Then, to get stuck into the testing device grips, both specimen ends were glued into aluminum rings using cyanoacrylate adhesive. Specimen images were taken with digital video camera disposed in an optical microscope (ZEISS). The image resolution was $4 \mu \mathrm{m} /$ pixel. The specimen geometry was measured using a custom imaging analysis software. A multi-threshold technique was associated with a mean square method to obtained the main size in the radial direction, $L_{r}$, and in the vertical direction, $L_{z}$. In Fig. (4), one can clearly observes the fibers orientation on the left image and the successive lamellae on the right one. Finally, 12 porcine and 6 lamb specimens were prepared to analyze the unidimensional mechanical behavior of annulus along the circumferential direction $\overrightarrow{e_{\theta}}$.

\section{Mechanical testing}

Prior to testing, the specimens were stored in a $0.15 \mathrm{Mol} / \mathrm{l} \mathrm{NaCl}$ solution at $\mathrm{T}=37^{\circ} \mathrm{C}$. The testing device was composed of a Texture Analyzer (TA-XT2, Stable Micro Systems, UK) with a $50 \mathrm{~N}$ load cell. Each sample was placed into the testing device grips and stretched during 5 
loading cycles from 0 to $1 \mathrm{~mm}$ lengthening in order to reach a maximum strain of $\varepsilon_{\max }=10 \%$. The extension rate was $0.01 \mathrm{~mm} / \mathrm{s}$ and the tension load was recorded every $0.01 \mathrm{~s}$.

\section{Data and statistical analysis}

All statistical analysis were realized with the GRETL software ${ }^{33}$. The significance $p$-value is obtained with a Student paired $t$-test. A value of $p<0.05$ indicates a significant difference while a value of $p>0.05$ means a negligible difference.

\section{Results}

Stress-strain curves from uniaxial tensile tests are presented first, then the ability of the rheological model to represent the experimental behavior is addressed and finally, the geometrical implications of this model is analyzed.

\section{Cyclic uniaxial tensile tests}

The whole set of annulus samples were subjected to the experimental protocol described in subsection Mechanical testing. Concerning two annulus samples, the evolution of the circumferential tensile stress as a function of strain is plotted in Fig. (5). Only the first two cycles are presented here. These results exhibit a strong non-linear behavior with a rigidity increase as the stress increases. Similar observations have been done with all investigated specimens. This particular behavior leads to the stiffening of the intervertebral disc tissue for large strains. Concerning the loading cycle, a significant hysteresis is observed. This features an energy dissipation measured by the area inside one cycle. This agrees with the essential dissipative function of intervertebral discs. In the second cycle, the dissipated energy is systematically lower. The successive loading cycles generally converge towards a single curve as already shown by Tower et al. ${ }^{31}$.

\section{Mechanical behaviors}

From these experimental results, the physical properties associated with the rheological model, i.e., the initial fibers orientation, $\theta_{0}$, and the mechanical characteristics, $\left(E_{f}, E_{m}\right.$ and $\left.\eta_{m}\right)$, are computed using a Levenberg-Marquardt identification algorithm. Concerning two samples, the rheological model is compared to the first experimental loading cycle in Fig. (6). A fairly good agreement is obtained since our rheological model can accurately describe the stress-strain curve along the whole cycle. 


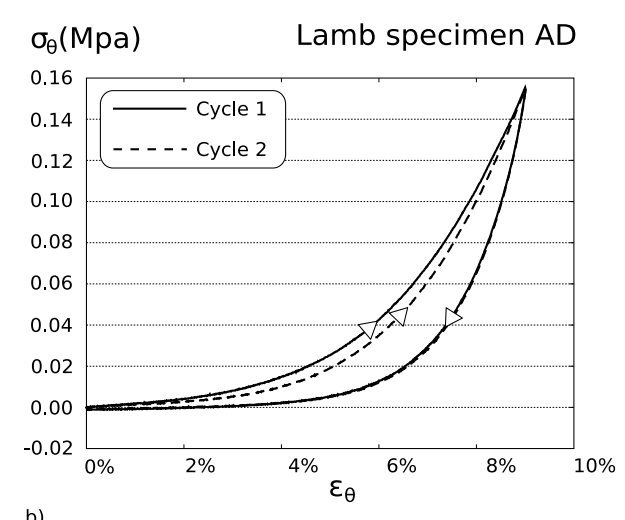

b)
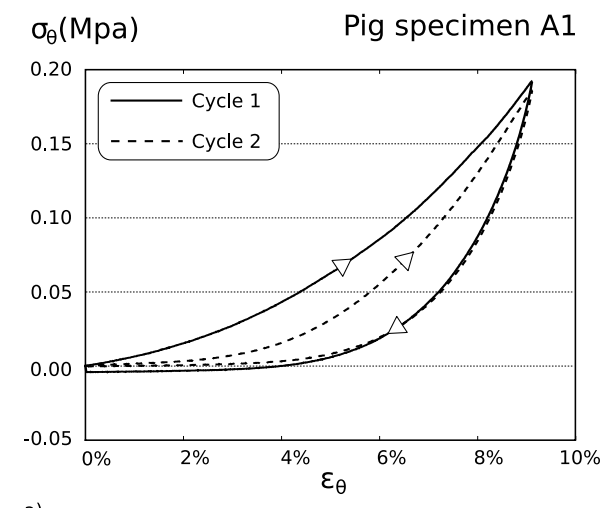

a)

Figure 5: Experimental curves of the stress versus strain: a) pig specimen, b) lamb specimen. 

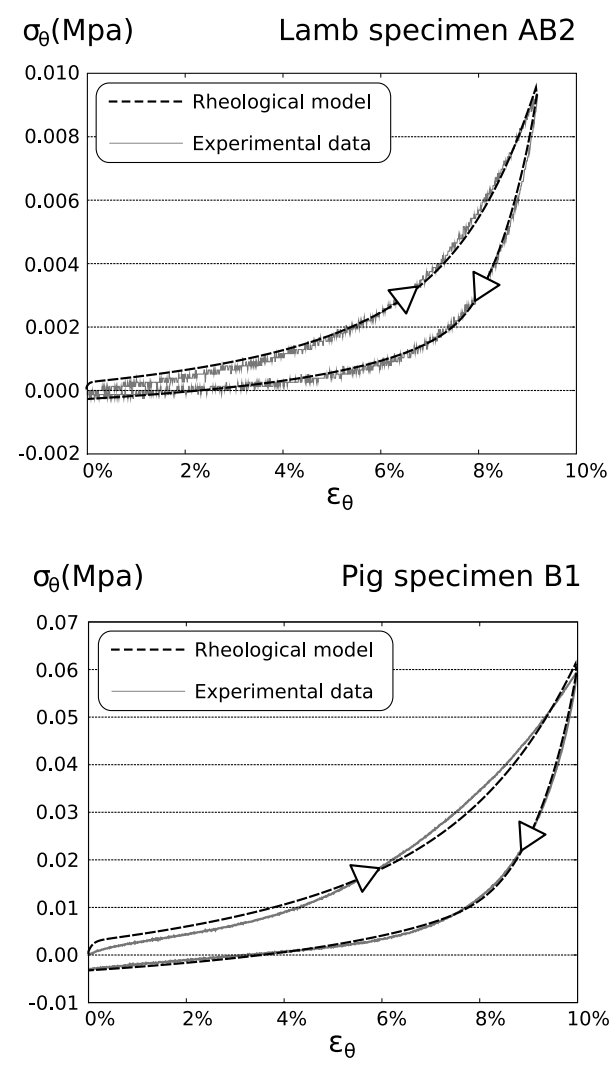

Figure 6: First stress-strain cycle curve of a) a pig specimen, b) a lamb specimen. Comparison between experimental measures and computed behavior. 
Table 1: Mechanical properties of pig and lamb specimens identified from experimental data (average[confidence interval at 95\%]).

\begin{tabular}{|c|c|c|c|c|c|}
\hline & $E_{f}(\mathrm{Mpa})$ & $E_{m}(\mathrm{Mpa})$ & $\eta_{m}($ Mpa.s $)$ & $\theta_{0}\left({ }^{\circ}\right)$ & $R^{2}$ \\
\hline \multicolumn{6}{|c|}{ Lamb specimen } \\
\hline$(\mathrm{n}=6)$ & $12.3[-7.1 ; 31.8]$ & $0.023[-0.01 ; 0.056]$ & $0.59[-1.49 ; 2.68]$ & $22.89[21.58 ; 24.20]$ & $0.995[0.99 ; 0.998]$ \\
\hline \multicolumn{6}{|l|}{ Pig specimen } \\
\hline$(\mathrm{n}=12)$ & $6.6[3.3 ; 9.8]$ & $0.0191[0.009 ; 0.028]$ & $0.61[0.25 ; 0.97]$ & $22.01[20.69 ; 23.33]$ & $0.995[0.993 ; 0.997]$ \\
\hline Total $(\mathrm{n}=18)$ & $8.3[3.4 ; 13]$ & $0.0196[0.01 ; 0.028]$ & $0.6[0.3 ; 0.91]$ & $22.27[21.32 ; 23.22]$ & $0.995[0.993 ; 0.996]$ \\
\hline
\end{tabular}

The physical characteristics identified with all specimens are summarized in Tab. (1). The fitting quality is evaluated based on the correlation factor, $R^{2}$, showing a very good accuracy in each cases. Regarding to the mechanical parameters, the confidence intervals reveal high variabilities of the identified properties. This can partly be attributed to some sample geometry discrepancies and is discussed in the last section. However, when focusing on the initial fibers orientation, high-confidence values have been obtained without significant differences between pig and lamb samples $(p=0.38)$.

\section{Model results}

To estimate the efficiency of this rheological model, the mechanical behavior of two annulus samples have been simulated using the physical characteristics identified in the previous section. The characteristics of the pig specimen are $\left(E_{f}=6.6 \mathrm{Mpa}, E_{m}=0.0191 \mathrm{Mpa}, \eta_{m}=0.61 \mathrm{Mpa} . \mathrm{s}, \theta_{0}=22.01^{\circ}\right)$, while the lamb specimen ones are $\left(E_{f}=12.3 \mathrm{Mpa}, E_{m}=0.023 \mathrm{Mpa}, \eta_{m}=0.59 \mathrm{Mpa} . \mathrm{s}, \theta_{0}=22.89^{\circ}\right)$. In Fig. (), three plots are presented. The first one represents the mechanical behavior of both samples during the first loading cycle (Fig. a), emphasizing the strong non-linear behavior. The tension / compression asymmetrical behavior must be noticed since the computed stiffness is lower in compression than in traction as already shown by Wagner and Lotz ${ }^{32}$. This particular behavior mainly results from the reorientation of collagen fibers along the traction direction. This is represented by the evolution of the fibers angle, $\theta$, as a function of strain, $\varepsilon_{\theta}$, in Fig. (b). The reorientation rate, defined by the average slope of this plot, is roughly constant and ranges between $-1.7^{\circ} / \%$ and $-2.0^{\circ} \%$. Indeed, as the fibers get oriented along the loading direction, $\overrightarrow{e_{\theta}}$, they participate more in the sample rigidity that leads to a global stiffness increase. The major role of fibers reorientation is confirmed when focusing on the fibers strain, $\varepsilon_{f}$, plotted in Fig. (c) as a function of the circumferential strain, $\varepsilon_{\theta}$. This shows that the collagen fibers extension is 
relatively low with a maximal extension of $2 \%$. In compression, the orientation angle is large and the fibers are weakly stressed.

\section{Discussion}

The microstructural informations gathered in this work agree with previous researches concerning the global water content ${ }^{22,21}$ and the collagen fibers size ${ }^{28,19}$. The significant differences observed between pig and lamb samples mainly results from the ages difference (lamb: 6 months, pig: 12 months). This is confirmed by the stronger mechanical characteristics observed with pig samples than those obtained with lamb ones.

The experimental unidimensional tensile tests reveal classical features of the annulus mechanical behavior, i.e., a non-linear behavior with stiffness increasing, an hysteretic evolution under loading cycle and a mechanical adaptation beyond the second loading cycle. These hysteretic cycles are associated with the visco-elastic nature of annulus tissue ${ }^{18}$. It gives prominence to the coupling between the global mechanical behavior and the viscous flow through the matrix that characterizes of poro-elastic media. Indeed, the visco-elastic properties of annulus tissue should be related to its liquid permeability ${ }^{17,26,34}$.

Between the different specimens tested, some discrepancies, revealed by the large confidence intervals given in Tab. (1), are observed. Some possible reasons are enumerated below.

The low number of specimens under investigation do not allow to identify the rheological parameters with confidence. This aspect is emphasized in the case of lamb where only 6 samples were tested, while the 12 pig samples lead to a better estimation.

Moreover, the mechanical behavior of annulus tissue highly depends on the localization inside the $\operatorname{disc}^{4,36}$. Indeed, the macroscopic mechanical characteristics are governed by the fibers orientation while it ranges from $\approx 25^{\circ}$ in outer annulus lamellae to $\approx 45^{\circ}$ close to the nucleus ${ }^{6,25,32}$. Meanwhile, the collagen fibers density increases in external annulus tissue ${ }^{25}$. These microstructural and composition variations affect significantly the reproducibility of experimental tests since the sampling is not sufficiently precise with respect to the localization.

Eventually, annulus samples were realized with a $2 \times 2 \mathrm{~mm}$ cross-section. In the radial direction, $\overrightarrow{e_{r}}$, a sample includes several lamellae (Fig. 4b). Since the viscous flow through the matrix influences the sample global stiffness, the number of lamellae acts as a characteristic size in the visco-elastic behavior by governing the liquid pressure relaxation time in the radial direction. Therefore, unixial tensile tests do not bring up the intrinsic mechanical behavior of one lamella, but rather provide the macroscopic behavior of an heterogeneous anisotropic structure ${ }^{15}$. Since 

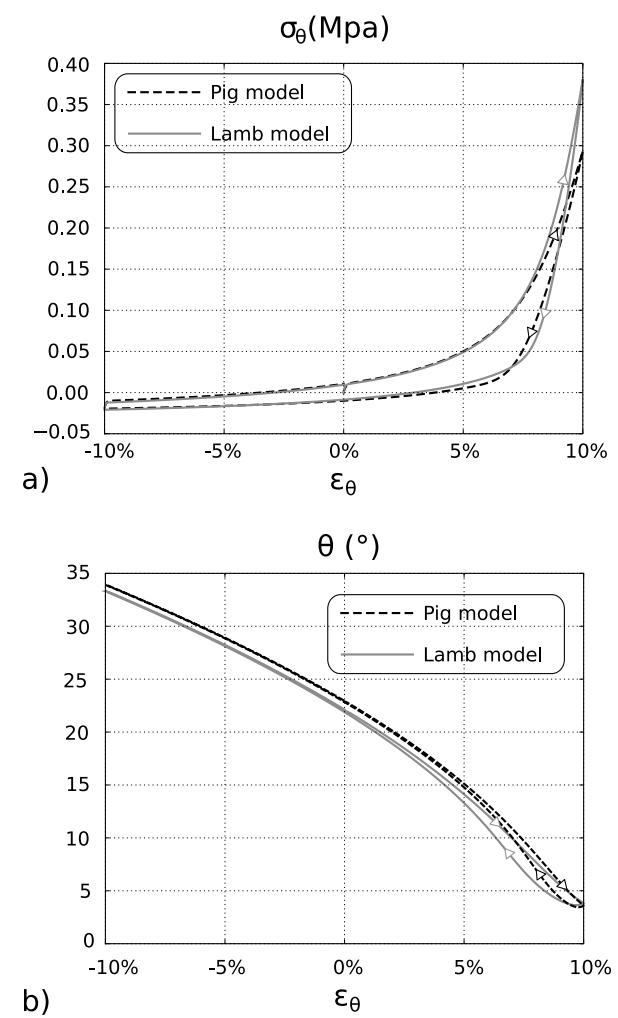

b)

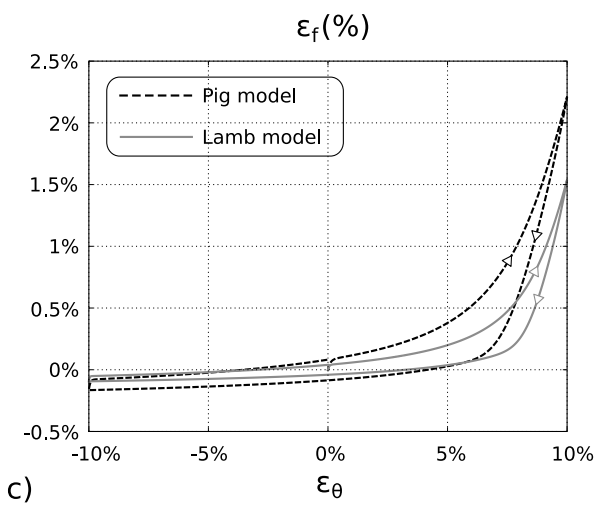

Figure 7: Computed mechanical behaviors of two annulus samples subjected to one loading traction-compression cycle: a) stress-strain curve, b) fibers orientation, c) fibers strain. 
the number of lamellae differs from one sample to another, this aspect could explain the observed discrepancies.

With a low number of parameters, 3 mechanical and 1 geometrical characteristics, this rheological model is able to represent several features of the mechanical behavior of annulus fibrosus tissue, i.e., non-linear stiffness, hysteretic visco-elastic behavior, reorientation of collagen fibers while loading and anisotropy in the $\left(\overrightarrow{e_{\theta}} ; \overrightarrow{e_{z}}\right)$ plan. It should be noticed that, with this model, the strong non-linear behavior is obtained only for a limited range of the initial fibers angle, $\theta_{0} \approx 23^{\circ}$. This is confirmed by the small confidence interval computed when identifying the initial fibers angle from experimental measures (Tab. 1). This initial angle is close to the fibers angle directly measured on outer annulus tissue, $\theta_{0} \approx 25^{\circ 6}$. With a larger fibers orientation angle, as observed with inner annulus tissue, the mechanical behavior is more linear ${ }^{3,32}$. However, our tensile tests have been performed without preconditioning as usually done in previous experimental works ${ }^{11,3,13}$. As reported by Tower et al. ${ }^{31}$, a transition occurs when the collagen fibers become uncrimped. By imaging techniques, they have shown that a significant fibers reorientation takes place during the preconditioning and depends on the choice of the reference configuration. Thus, in our case, not taking into account this transition could affect the quality of identified properties and leads to under-estimate the fibers initial angle. Anyway, this heuristic rheological model brings out that the fibers reorientation can account solely for the strong non-linear behavior without relying on the assumption that the stiffening results from a transition between crimped and uncrimped fibers ${ }^{16}$.

Therefore, we assess that the non-linear behavior mainly results from the collagen fibers reorientation. This point of view relies on the strong microstructural assumption that the fibers reorientation is governed by the macroscopic strain, i.e., there is no relative displacement between the fibers and the matrix. This leads to a fibers reorientation rate ranging between $-1.7^{\circ} / \%$ and $-2.0^{\circ} / \%$ (Fig. b). This rate is about 2 times larger than direct measures based on imaging techniques proposed by Guerin and Elliott ${ }^{13}$, which seems to indicate that this microstructural assumption is not completely efficient.

Concerning Young's modulus of the collagen fibers, the average estimated value, $E_{f}=8.27$ $\mathrm{Mpa}$, is in agreement with already published results for low fibers strain, $\varepsilon_{f} \leq 2 \%^{15,29}$. In these conditions, the elastic linear assumption is therefore a fair representation of fibers mechanical behavior ${ }^{9}$. Regarding to the matrix stiffness, $E_{m}$, the values presented in this work are about 20 times less than previous experimental results ${ }^{19,36,29}$. However, these characteristics should not be compared to each other since they do not have the same physical meaning. So far, the matrix has been described using only one stiffness while we proposed a visco-elastic behavior based on two 
properties, a stiffness, $E_{m}$, and a viscosity, $\eta_{m}$. Thus, matrix stresses are partly supported by the viscous flow leading to a lower matrix stiffness. To check this proposition, tensile tests must be carried out with different extension velocity (Eq. 3) and this is the scope of future work.

Relying on such description of the poro-mechanical behavior will have strong implications on the coupling between strains and fluid transfers. In particular, this kind of woven oriented structure can exhibit, at the macroscopic scale, values of Poisson's coefficients that lie outside of the classical range valid for homogeneous materials, $0<\nu<0.5$. This unusual behavior mainly depends on the fibers orientation angle, $\theta$. With angles around $25^{\circ}$, as observed with annulus tissue, the macroscopic transverse shrinkage is larger than the longitudinal extension. In the case of uniaxial tension, this leads to a negative total volume variation that cannot be recovered with classical poro-elastic formulations usually used ${ }^{4,2,24}$. Instead of a global swelling, the matrix shrinkage generates an additional fluid pressure and an outward liquid flow.

Therefore, when focusing on the cell nutrients transfer mechanisms from the vertebrae toward the nucleus under cyclic loading, these particular coupling effects significantly influence liquid flows. It is therefore a major issue to develop an accurate poro-mechanical formulation in order to propose a comprehensive model of the cell nutrition in the intervertebral disc.

\section{Acknowledgments}

This research was supported by the C.N.R.S (Projets Exploratoires Pluridisciplinaires) and the University of Montpellier II.

\section{References}

[1] Accadbled, F., Ambard, D., de Gauzy, J. S., Swider, P., 2008. A measurement technique to evaluate the macroscopic permeability of the vertebral end-plate. Med Eng Phys 30, 116-122.

[2] Argoubi, M., Shirazi-Adl, A., 1996. Poroelastic creep response analysis of a lumbar motion segment in compression. J Biomech 29, 1331-1339.

[3] Bass, E. C., Ashford, F. A., Segal, M. R., Lotz, J. C., 2004. Biaxial testing of human annulus fibrosus and its implications for a constitutive formulation. Ann Biomed Eng 32, 1231-1242.

[4] Best, B. A., Guilak, F., Setton, L. A., Zhu, W., Saed-Nejad, F., Ratcliffe, A., Weidenbaum, M., Mow, V. C., 1994. Compressive mechanical properties of the human annulus fibrosus and their relationship to biochemical composition. Spine 19, 212-221. 
[5] Bruehlmann, S. B., Hulme, P. A., Duncan, N. A., 2004. In situ intercellular mechanics of the bovine outer annulus fibrosus subjected to biaxial strains. J Biomech 37, 223-231.

[6] Cassidy, J. J., Hiltner, A., Baer, E., 1989. Hierarchical structure of the intervertebral disc. Connect Tissue Res 23, 75-88.

[7] Costi, J. J., Stokes, I. A., Gardner-Morse, M., Laible, J. P., Scoffone, H. M. Iatridis, J. C., 2007. Direct measurement of intervertebral disc maximum shear strain in six degrees of freedom: Motions that place disc tissue at risk of injury. Journal of Biomechanics 40, 2457-2466.

[8] Elliott, D. M., Setton, L. A., 2001. Anisotropic and inhomogeneous tensile behavior of the human anulus fibrosus: experimental measurement and material model predictions. Journal of Biomechanical Engineering, 123, 256-63.

[9] Eppell, S. J., Smith, B. N., Kahn, H., Ballarini, R., 2006. Nano measurements with microdevices: mechanical properties of hydrated collagen fibrils. Journal of the Royal Society Interface $3,117-121$.

[10] Ferguson, S. J., Ito, K., Nolte, L. P., 2004. Fluid flow and convective transport of solutes within the intervertebral disc. J Biomech 37 (2), 213-221.

[11] Fujita, Y., Duncan, N. A., Lotz, J. C., 1997. Radial tensile properties of the lumbar annulus fibrosus are site and degeneration dependent. J Orthop Res 15, 814-819.

[12] Grunhagen, T., Wilde, G., Soukane, D. M., Shirazi-Adl, S. A., Urban, J. P. G., 2006. Nutrient supply and intervertebral disc metabolism. J Bone Joint Surg Am 88 Suppl 2, 30-35.

[13] Guerin, H. A. L., Elliott, D. M., 2006. Degeneration affects the fiber reorientation of human annulus fibrosus under tensile load. J Biomech 39, 1410-1418.

[14] Guerin, H. A. L.,Elliott, D. M., 2007. Quantifying the Contributions of Structure to Annulus Fibrosus Mechanical Function Using a Nonlinear, Anisotropic, Hyperelastic Model. J Orthop Res $25,508-516$.

[15] Holzapfel, G. A., Schulze-Bauer, C. A. J., Feigl, G., Regitnig, P., 2005. Single lamellar mechanics of the human lumbar annulus fibrosus. Biomech Model Mechanobiol 3, 125-140.

[16] Holzapfel, G. A., Cacho, F., Elbischger, P., Rodriguez, J., Doblare, M., 2007. A constitutive model for fibrous tissues considering collagen fiber crimp. International Journal of Non-Linear Mechanics 42, 391-402. 
[17] Iatridis, J. C., Setton, L., Weidenbaum, M., Mow, V. C., 1997. The viscoelastic behavior of the non-degenerate human lumbar nucleus pulposus in shear. Journal of Biomechanics 30, 1005-1013.

[18] Iatridis, J. C., Kumar, S., Foster, R. J., Weidenbaum, M. and Mow, V. C., 2002. Shear mechanical properties of human lumbar annulus fibrosus. Journal of Orthopaedic Research 17, $732-737$.

[19] Iatridis, J. C., ap Gwynn, I., 2004. Mechanisms for mechanical damage in the intervertebral disc annulus fibrosus. Journal of Biomechanics 37, 1165-1175.

[20] Iatridis, J. C., MacLean, J. J., Ryan, D. A., 2005. Mechanical damage to the intervertebral disc annulus fibrosus subjected to tensile loading. Journal of Biomechanics 38, 557-565.

[21] Iatridis, J. C., MacLean, J. J., O’Brien, M., Stokes, I. A. F., 2007. Measurements of proteoglycan and water content distribution in human lumbar intervertebral discs. Spine 32, 1493-1497.

[22] Johnstone, B., Urban, J. P., Roberts, S., Menage, J., 1992. The fluid content of the human intervertebral disc. comparison between fluid content and swelling pressure profiles of discs removed at surgery and those taken postmortem. Spine 17, 412-416.

[23] Klisch, S. M., Lotz, J. C., 1999. Application of a fiber-reinforced continuum theory to multiple deformations of the annulus fibrosus. J Biomech 32, 1027-1036.

[24] Magnier, C., Boiron, O., Wendling-Mansuy, S., Chabrand, P., Deplano, V., 2009. Nutrient distribution and metabolism in the intervertebral disc in the unloaded state: A parametric study. Journal of Biomechanics 42, 100-108.

[25] Marchand, F., Ahmed, A. M., 1990. Investigation of the laminate structure of lumbar disc annulus fibrosus. Spine 15, 402-410.

[26] Riches, P. E., Dhillon, N., Lotz, J. C., Woods, A. W., McNally, D. S., 2002. The internal mechanics of the intervertebral disc under cyclic loading. J Biomech 35, 1263-1271.

[27] Shirazi-Adl, S. A., 1989. Strain in fibers of a lumbar disc. analysis of the role of lifting in producing disc prolapse. Spine 14, 96-103.

[28] Skaggs, D. L., Weidenbaum, M., Iatridis, J. C., Ratcliffe, A., Mow, V. C., 1994. Regional variation in tensile properties and biochemical composition of the human lumbar annulus fibrosus. Spine 19, 1310-1319. 
[29] Smith, L. J., Byers, S., Costi, J. J., Fazzalari, N.L., 2008. Elastic Fibers Enhance the Mechanical Integrity of the Human Lumbar Anulus Fibrosus in the Radial Direction. Annals of Biomedical Engineering 36, 214-223.

[30] Soukane, D. M., Shirazi-Adl, S. A., Urban, J. P. G., 2007. Computation of coupled diffusion of oxygen, glucose and lactic acid in an intervertebral disc. J Biomech 40, 2645-2654.

[31] Tower, T. T., Neidert, M. R., Tranquillo, R. T., 2002. Fiber alignment imaging during mechanical testing of soft tissues. Ann Biomed Eng 30, 1221-1233.

[32] Wagner, D. R., Lotz, J. C., 2004. Theoretical model and experimental results for the nonlinear elastic behavior of human annulus fibrosus. J Orthop Res 22, 901-909.

[33] Yalta, A. T., Yalta, A. Y., 2007. Gretl 1.6.0 and its numerical accuracy. Journal of Applied Econometrics 22, 849-854.

[34] Yao, H., Justiz, M.A., Flagler, D. and Gu, W.Y., 2002. Effects of Swelling Pressure and Hydraulic Permeability on Dynamic Compressive Behavior of Lumbar Annulus Fibrosus. Annals of Biomedical Engineering 30, 1234-1241.

[35] Yao, H., Gu, W. Y., 2007. Three-dimensional inhomogeneous triphasic finite-element analysis of physical signals and solute transport in human intervertebral disc under axial compression. J Biomech 40, 2071-2077.

[36] Yin, L., Elliott, D. M., 2005. A homogenization model of the annulus fibrosus. J Biomech 38, $1674-1684$.

[37] Yu, J., Tirlapur, U., Fairbank, J., Handford, P., Roberts, S., Winlove, C. P., Cui, Z., Urban, J. P. G., 2007. Microfibrils, elastin fibres and collagen fibres in the human intervertebral disc and bovine tail disc. J Anat 210, 460-471. 